\title{
Effects of initiating moderate wine intake on abdominal adipose tissue in adults with type 2 diabetes: a 2 -year randomized controlled trial
}

Rachel Golan ${ }^{1, *}$, llan Shelef ${ }^{2}$, Elad Shemesh ${ }^{2}$, Yaakov Henkin ${ }^{2}$, Dan Schwarzfuchs ${ }^{3}$, Yftach Gepner ${ }^{1}$, llana Harman-Boehm ${ }^{2}$, Shula Witkow ${ }^{1}$, Michael Friger ${ }^{1}$, Yoash Chassidim ${ }^{2}$, Idit F Liberty ${ }^{2}$, Benjamin Sarusi ${ }^{3}$, Dana Serfaty ${ }^{1}$, Nitzan Bril ${ }^{1}$, Michal Rein ${ }^{1}$, Noa Cohen ${ }^{1}$, Sivan Ben-Avraham ${ }^{1}$, Uta Ceglarek ${ }^{4}$, Michael Stumvoll ${ }^{4}$, Matthias Blüher ${ }^{4}$, Joachim Thiery ${ }^{4}$, Meir J Stampfer ${ }^{5}$, Assaf Rudich ${ }^{1}$ and Iris Shai ${ }^{1}$

'Department of Public Health, Faculty of Health Sciences, Ben-Gurion University of the Negev, PO Box 653, Beer-Sheva 84 105, Israel: ${ }^{2}$ Soroka University Medical Center, Beer-Sheva, Israel: ${ }^{3}$ Nuclear Research Center Negev, Dimona, Israel: ${ }^{4}$ Department of Medicine, University of Leipzig, Leipzig, Germany: ${ }^{5}$ Channing Division of Network Medicine, Department of Medicine, Brigham and Women's Hospital and Harvard School of Public Health, Boston, MA, USA

Submitted 28 December 2015: Final revision received 8 August 2016: Accepted 16 August 2016: First published online 3 0ctober 2016

\begin{abstract}
Objective: To generate evidence-based conclusions about the effect of wine consumption on weight gain and abdominal fat accumulation and distribution in patients with type 2 diabetes.

Design: In the 2-year randomized controlled CASCADE (CArdiovaSCulAr Diabetes \& Ethanol) trial, patients following a Mediterranean diet were randomly assigned to drink $150 \mathrm{ml}$ of mineral water, white wine or red wine with dinner for 2 years. Visceral adiposity and abdominal fat distribution were measured in a subgroup of sixty-five participants, using abdominal MRI.

Setting: Ben-Gurion University of the Negev, Soroka-Medical Center and the Nuclear Research Center Negev, Israel.

Subjects: Alcohol-abstaining adults with well-controlled type 2 diabetes.

Results: Forty-eight participants (red wine, $n$ 27; mineral water, $n$ 21) who completed a second MRI measurement were included in the 2-year analysis. Similar weight losses (SD) were observed: red wine $1.3(3.9) \mathrm{kg}$; water $1.0(4.2) \mathrm{kg}$ $(P=0.8$ between groups). Changes $(95 \% \mathrm{CI})$ in abdominal adipose-tissue distribution were similar: red wine, visceral adipose tissue (VAT) $-3 \cdot 0(-8 \cdot 0,2 \cdot 0)$ $\%$, deep subcutaneous adipose tissue (DSAT) $+5 \cdot 2(-1 \cdot 1,11 \cdot 6) \%$, superficial subcutaneous adipose tissue (SSAT) $-1 \cdot 9(-5 \cdot 0,1 \cdot 2) \%$; water, VAT $-3 \cdot 2(-8 \cdot 9,2 \cdot 5)$ $\%$, DSAT $+2 \cdot 9(-2 \cdot 8,8 \cdot 6) \%$, SSAT $-0 \cdot 15(-3 \cdot 3,2 \cdot 9) \%$. No changes in antidiabetic medication and no substantial changes in energy intake $(+126$ (SD 2889) kJ/d $(+30 \cdot 2(\mathrm{sD} 690) \mathrm{kcal} / \mathrm{d}), P=0 \cdot 8)$ were recorded. A 2 -year decrease in glycated $\mathrm{Hb}$ $(\beta=0.28, P=0.05)$ was associated with a decrease in VAT.

Conclusions: Moderate wine consumption, as part of a Mediterranean diet, in persons with controlled diabetes did not promote weight gain or abdominal adiposity.
\end{abstract}

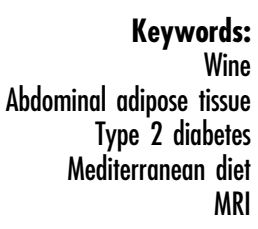

The effect of alcohol consumption on the accumulation and distribution of abdominal fat is controversial. While some studies report a positive association between alcohol consumption and the risk of central obesity ${ }^{(1,2)}$, others suggest an inverse association ${ }^{(3-5)}$ or no association ${ }^{(6,7)}$. In patients with type 2 diabetes, the effect of moderate alcohol consumption on abdominal fat distribution had not yet been assessed systematically until very recently ${ }^{(8)}$, but further analysis is still required and is reported in the current paper.

Visceral adipose tissue (VAT) accumulation, which has been strongly associated with cardiometabolic risks ${ }^{(9-12)}$, differs anatomically and functionally from the peripheral (lower body) and abdominal subcutaneous adipose tissue (SAT) ${ }^{(13)}$. 
Patients with type 2 diabetes tend to have more VAT and less SAT than people without type 2 diabetes ${ }^{(14)}$, which corresponds to their increased risk for atherosclerosis and $\mathrm{CVD}^{(15)}$. Recently, we suggested that increased absolute and relative distribution of fat in the abdominal superficial SAT sub-depot in patients with type 2 diabetes may signify a more favourable cardiometabolic risk profile ${ }^{(16)}$.

After a 3-month feasibility trial ${ }^{(17)}$ in 109 patients with type 2 diabetes, we initiated the 2-year CASCADE (CArdiovaSCulAr Diabetes \& Ethanol) trial $^{(8)}$ in 224 patients with type 2 diabetes. We hypothesized that initiating moderate wine consumption would decrease cardiometabolic risk. In the subgroup described in the current paper, we aimed to address the effect of initiating wine consumption on the accumulation and distribution of central adiposity, as evaluated by abdominal MRI.

\section{Methods}

\section{Eligibility and study design}

The participants in the 2-year CASCADE trial comprised alcohol-abstaining persons with diabetes, diagnosed according to the American Diabetes Association criteria, and aged 40-75 years. Participant characteristics were measured at baseline and the participants were randomly assigned to one of three groups in a parallel design (1:1:1), namely with mineral water, white wine or red wine, $150 \mathrm{ml}$, at dinnertime, as described below. The study design has been reported in detail ${ }^{(8)}$. All participants provided informed consent and the study was approved and monitored by the Human Subjects Committees of the Soroka University Medical Center and the Ben-Gurion University of the Negev. No financial compensation was provided to the participants.

\section{Intervention}

Participants in the CASCADE trial were randomized to one of three groups: (i) dry red wine (Golan Heights Winery; $16.9 \mathrm{~g}$ of ethanol $(14.2 \%$ by volume), with $270.1 \mathrm{mg}$ of gallic acid equivalent of total phenols; $502 \mathrm{~kJ}$ (120 kcal)/ $150 \mathrm{ml}$ ); (ii) dry white wine (Golan Heights Winery; $15.8 \mathrm{~g}$ of ethanol ( $13.3 \%$ by volume), with $38.5 \mathrm{mg}$ of gallic acid equivalent of total phenols; $464 \mathrm{~kJ}(111 \mathrm{kcal}) / 150 \mathrm{ml})$; or (iii) mineral water (Mey Eden). Participants were instructed to consume $150 \mathrm{ml}$ ( 5 fluid ounces; measured in a standard 150-ml measuring glass provided by the study) of the relevant beverage with dinner. The participants assigned to consume wine were instructed to increase their wine intake gradually over the first few weeks and to avoid driving after dinner. All beverages were freely provided. We assessed adherence to beverage consumption by keeping track of the returned bottles and requiring the participants complete a specific questionnaire about their alcohol intake within or outside the study protocol.

With the aim to achieve a comparable healthy diet and to provide an incentive to participate, we provided all participants with guidelines to follow a Mediterranean diet, regardless of their previous dietary habits. Group sessions were held for all participants with clinical dietitians at 1-month intervals for the first three months and at 3-month intervals thereafter. Dietary consumption was monitored by a validated $\mathrm{FFQ}^{(18,19)}$ at baseline, after 6 months and at the end of the study. Dietary guidelines were based on quality rather than quantity of foods. Participants in the wine groups were instructed to reduce their daily allowance by $418 \mathrm{~kJ}$ ( $100 \mathrm{kcal})$ to compensate for the energy added from the wine. We made no other attempts to restrict energy intake.

At 0,6 and 24 months, participants completed validated $^{(20)}$ electronic questionnaires, which collected data on demographics, lifestyle patterns, specific medications, symptoms and quality of life. The quality of life questionnaire included questions regarding the participants' feelings, sleeping habits and bowel movements, on a weekly basis. Exercise habits were evaluated using the metabolic equivalent of task (MET) score.

Of the 224 participants in the CASCADE trial, a subgroup of seventy-three men and women (mainly from the red wine and water groups; see online supplementary material, Supplemental Fig. 1) underwent the same MRI protocol study of the abdomen at baseline and after 2 years, as previously described ${ }^{(15)}$. The randomization for the entire CASCADE study ${ }^{(8)}$ included the additional subgroup analysis of those who participated in this sub-study. Of the above-described seventy-three participants in the MRI study, forty-eight (red wine, $n 27$; mineral water, $n$ 21) completed the second MRI measurement and were thus included in the 2-year analysis presented in the current paper.

MRI scans were assessed using a Matlab-based in-house program. Adipose tissues of specific anatomical landmarks were quantified and colour-coded. The analysis was blind to the time point (pre- or post-intervention) and to treatment assignment. Both absolute abdominal fat mass and fat distribution were calculated using the average of three MRI slices, at levels of L2-L3, L5-L4 and L5-S1 inter-vertebrae space, as recommended previously ${ }^{(21,22)}$. Measurements at baseline and after 2 years allowed calculation of the changes in fat distribution during the trial. Participants were weighed without shoes to the nearest $0.1 \mathrm{~kg}$. Waist circumference was measured halfway between the last rib and the iliac crest. Blood samples were obtained at 0, 6 and 24 months at 08.00 hours after an $8 \mathrm{~h}$ fast, stored at $-80^{\circ} \mathrm{C}$ and analysed in one batch at the end of the study at the Leipzig laboratories, Germany. The clinic and laboratory staff members were unaware of the treatment assignments, and the study coordinators were unaware of all outcome data until the end of the intervention. 


\section{Statistical analysis}

Our primary end points were total abdominal adipose tissue and abdominal adipose tissue distribution. We analysed the data in their raw values without transformations. We used both absolute and proportional fat distribution for the analysis to account for sub-depot adiposity and inter-depot distribution, respectively. Fat distribution was calculated by dividing each fat depot by total abdominal adipose tissue, thereby creating three new variables: superficial SAT (SSAT\%), deep SAT (DSAT\%) and VAT\%. Since there are significant differences in the content of phenols in red and white wines, and since only eight participants from the white wine group of the CASCADE study underwent an MRI, we excluded these participants from our analysis. The presented analysis therefore includes two intervention groups, red wine ( $n$ 27) and mineral water ( $n$ 21). The main results are presented as changes from baseline in the wine group compared with changes from baseline in the water group and the corresponding $95 \%$ confidence intervals. Changes from baseline were calculated and compared between groups by ANOVA using the Studentized range test. The non-parametric Wilcoxon test was used to compare the values within each of the two groups, and Pearson's and Spearman's rho correlations were used to evaluate associations between changes in absolute and proportional fat depots and changes in fasting blood biomarkers, clinical parameters and dietary intake. Multivariate linear regression analyses, adjusted for intervention group, changes in BMI, sex and baseline VAT, were performed to evaluate variables associated with change in VAT after 2 years. We also performed similar multivariate linear regression analyses adjusted one at a time to the various classes of medical treatment used in this patient population (insulin therapy, oral hypoglycaemic medications, antihypertensive medications and lipid-lowering medications) and the same analyses stratified by sex. All $P$ values were two-sided. We used the statistical software packages Stata version 12 for randomization of participants and IBM SPSS Statistics Version 19.0 for all statistical analyses. Values reported are means and standard deviations unless otherwise stated.

\section{Results}

\section{Baseline characteristics}

Baseline characteristics were distributed similarly across the two study groups (Table 1) and were not different from those of the entire CASCADE cohort ${ }^{(8)}$. The participants (age 58 (range 40-73) years; 83\% men; glycated $\mathrm{Hb}\left(\mathrm{Hb}_{\mathrm{A} 1 \mathrm{c}}\right)$ level, $6.9 \%$; waist circumference, $104(\mathrm{SD} 9.8) \mathrm{cm}$ ) had an average of $44 \%$ abdominal visceral fat. Most participants were taking medications for diabetes, hypertension and/or hypercholesterolaemia. At baseline, weight $(r=0.5)$, BMI $(r=0.4)$ and waist circumference $(r=0.6)$ were all significantly associated with higher baseline VAT $(P<0.0001$ for all). Among the biomarkers, a higher baseline VAT was associated with decreased levels of HDL cholesterol $(r=-0 \cdot 3)$, apo A1 $(r=-0 \cdot 3)$ and adiponectin $(r=-0 \cdot 4)$, and with increased levels of fasting insulin $(r=0.4)$ and homeostatic model assessment of insulin resistance $(r=0.4 ; P<0.005$ for all). Similar associations were observed in an analysis of participants stratified by the use of oral glycaemic-control medications (data not shown). Visceral fat was not significantly associated with LDL cholesterol, apo B100, high-sensitivity C-reactive protein or IL-6 in this cohort.

\section{Two-year changes}

After 2 years, the participants exhibited modest and similar reductions in weight (red wine group, $-1 \cdot 3$ (95\% CI $-3 \cdot 6$, $0.8) \mathrm{kg}$; water group, -1.0 (95\% CI $-2.9,0.9) \mathrm{kg} ; P=0.8$ between groups) and increased HDL cholesterol (red wine group, +3.9 (sD 7.6) mg/dl; water group, +3.6 (sD 5.3) mg/dl; $P=0.8$ between groups). Forty-eight of the participants (red wine, $n$ 27; mineral water, $n$ 21) in the CASCADE study who completed a second MRI measurement formed the study population for this study. After 2 years, abdominal adipose tissue redistribution was observed in both groups. The values for the red wine group were: VAT $-3 \cdot 0$ (95\% CI $-8 \cdot 0,2 \cdot 0) \%(P=0 \cdot 2)$, DSAT $+5 \cdot 2(95 \% \mathrm{CI}-1 \cdot 1,11 \cdot 6) \%$ $(P=0 \cdot 1)$, SSAT -1.9 (95\% CI $-5 \cdot 0,1 \cdot 2) \%(P=0 \cdot 2)$. Those for the mineral water group were: VAT -3.2 (95\% CI -8.9 , $2 \cdot 5) \%(P=0 \cdot 2)$, DSAT $+2.9(95 \% \mathrm{CI}-2 \cdot 8,8 \cdot 6) \%(P=0 \cdot 3)$, SSAT $-0.15(95 \%$ CI $-3.3,2.9) \%(P=0.9$; Table 2$)$. These changes were not significantly different between the two groups and they were not significantly different from the baseline values. No significant differences in fat redistribution were found between men and women. No significant differences in weight loss or baseline characteristics were found between participants who completed the second MRI measurement and those who did not. As a follow-up to our baseline results ${ }^{(15)}$, we assessed the association between changes in abdominal adipose tissues and metabolic biomarkers. A univariate analysis showed that the 2-year decrease in VAT was associated with 2-year decreases in $\mathrm{Hb}_{\mathrm{A} 1 \mathrm{c}}(r=0 \cdot 5, P=0 \cdot 002)$, fasting glucose $(r=0 \cdot 3, P=0 \cdot 03)$ and BMI $(r=0.4, P=0.01)$ and 2-year increase in adiponectin $(r=-0 \cdot 4, P=0 \cdot 02)$. Of note, the 2 -year change in VAT was not associated with changes in waist circumference or with changes in systolic or diastolic blood pressure. In multivariate regression analyses adjusted for intervention group, sex and baseline VAT, and a 2-year change in BMI $(\beta=0.4, P=0 \cdot 13)$, a 2 -year decrease in $\mathrm{Hb}_{\mathrm{A} 1 \mathrm{c}}(\beta=0.4, \quad P=0.05)$ and a higher baseline VAT $(\beta=-0 \cdot 4, P=0 \cdot 01)$ remained significant independent variables associated with a decrease in VAT. (Homeostatic model assessment of insulin resistance and fasting glucose were not included since both variables were strongly 
Table 1 Baseline characteristics of the CASCADE sub-study population*

\begin{tabular}{|c|c|c|c|c|c|c|}
\hline \multirow[b]{2}{*}{ Variable } & \multicolumn{2}{|c|}{$\begin{array}{c}\text { Mineral water } \\
(n 29)\end{array}$} & \multicolumn{2}{|c|}{$\begin{array}{l}\text { Red wine } \\
\quad(n 36)\end{array}$} & \multicolumn{2}{|c|}{$\begin{array}{l}\text { Total } \\
(n 65)\end{array}$} \\
\hline & Mean & SD & Mean & SD & Mean & SD \\
\hline Age (years) & $57 \cdot 2$ & $7 \cdot 3$ & 59.1 & $7 \cdot 8$ & $58 \cdot 2$ & 7.5 \\
\hline Men $(\%)$ & $72 \cdot 4$ & & $91.7 \dagger$ & & 83.1 & \\
\hline Waist circumference $(\mathrm{cm})$ & $104 \cdot 1$ & 9.9 & $105 \cdot 2$ & 8.7 & 104.7 & $9 \cdot 2$ \\
\hline BMI $\left(\mathrm{kg} / \mathrm{m}^{2}\right)$ & 29.7 & 3.9 & $29 \cdot 3$ & 3.9 & 29.5 & 3.9 \\
\hline \multicolumn{7}{|l|}{ Blood pressure $(\mathrm{mmHg})$} \\
\hline Systolic & 133.7 & $13 \cdot 1$ & $137 \cdot 8 \dagger$ & $19 \cdot 7$ & $136 \cdot 0$ & $17 \cdot 3$ \\
\hline Diastolic & 78.5 & 8.8 & $78 \cdot 8$ & 11.8 & 78.4 & $10 \cdot 2$ \\
\hline \multicolumn{7}{|l|}{ Abdominal MRI (cm²) } \\
\hline Total subcutaneous AT & 2263.4 & 868.9 & 1995.4 & 652.5 & $2115 \cdot 0$ & $762 \cdot 3$ \\
\hline Superficial subcutaneous AT & $1077 \cdot 1$ & 561.9 & 919.9 & 379.9 & 990.0 & 472.5 \\
\hline Deep subcutaneous AT & $1186 \cdot 3$ & 477.6 & 1075.5 & 478.5 & 1124.9 & $477 \cdot 6$ \\
\hline Visceral AT & $1607 \cdot 6$ & 617.9 & $1910 \cdot 1$ & 974.6 & $1775 \cdot 9$ & 841.7 \\
\hline Total abdominal AT & $3967 \cdot 3$ & $1114 \cdot 6$ & $4022 \cdot 8$ & $1216 \cdot 5$ & 3998.5 & $1164 \cdot 2$ \\
\hline \multicolumn{7}{|l|}{ AT distribution, out of total AT (\%) } \\
\hline Superficial subcutaneous AT & $26 \cdot 8$ & 9.9 & 23.4 & 8.5 & 24.9 & $9 \cdot 2$ \\
\hline Deep subcutaneous AT & $29 \cdot 6$ & $8 \cdot 1$ & $27 \cdot 6$ & 11.2 & 28.5 & $9 . \overline{9}$ \\
\hline Visceral AT & $41 \cdot 2$ & $11 \cdot 2$ & $46 \cdot 0$ & 13.7 & 43.9 & $12 \cdot 8$ \\
\hline \multicolumn{7}{|l|}{ Glycaemic biomarkers } \\
\hline FPG (mg/dl) & $160 \cdot 3$ & $52 \cdot 0$ & $151 \cdot 1$ & 33.5 & 154.5 & $42 \cdot 1$ \\
\hline $\mathrm{Hb}_{\mathrm{A} 1 \mathrm{c}}(\%)$ & 7.0 & 1.2 & 6.9 & 1.0 & 6.9 & $1 \cdot 1$ \\
\hline HOMA-IR & $6 \cdot 0$ & 4.8 & 4.9 & 3.7 & $5 \cdot 4$ & $4 \cdot 2$ \\
\hline \multicolumn{7}{|l|}{ Lipid biomarkers } \\
\hline Total cholesterol (mg/dl) & $177 \cdot 3$ & 35.9 & $172 \cdot 2$ & $35 \cdot 4$ & 174.2 & $35 \cdot 2$ \\
\hline Serum TAG $(\mathrm{mg} / \mathrm{dl})$ & $187 \cdot 3$ & $181 \cdot 2$ & $135 \cdot 2$ & 68.4 & 157.5 & $130 \cdot 5$ \\
\hline Serum HDL-C (mg/dl) & 39.2 & 11.3 & 44.9 & 14.5 & $42 \cdot 2$ & $12 \cdot 8$ \\
\hline Serum LDL-C (mg/dl) & $100 \cdot 1$ & $30 \cdot 7$ & $97 \cdot 1$ & $32 \cdot 9$ & 98.5 & 31.5 \\
\hline Apo A1 (g/l) & 1.4 & 0.2 & 1.4 & 0.2 & 1.4 & 0.2 \\
\hline Apo B100 (q/l) & 1.0 & 0.2 & 0.9 & 0.2 & 0.9 & 0.2 \\
\hline \multicolumn{7}{|l|}{ Inflammatory biomarkers } \\
\hline Plasma hs-CRP (mg/l) & 4.4 & $5 \cdot 6$ & $4 \cdot 8$ & 8.4 & 4.7 & $7 \cdot 2$ \\
\hline IL-6 & 3.7 & 2.0 & $6 \cdot 8 \dagger$ & 6.9 & $5 \cdot 2$ & $5 \cdot 1$ \\
\hline \multicolumn{7}{|l|}{ Medications in current use $(n, \%)$} \\
\hline Oral glycaemic-control medications & 20 & $69 \cdot 0$ & 24 & $76 \cdot 7$ & 44 & $67 \cdot 7$ \\
\hline Insulin therapy & 3 & $10 \cdot 3$ & 6 & $16 \cdot 7$ & 9 & $13 \cdot 8$ \\
\hline Antihypertensive therapy & 16 & $55 \cdot 2$ & 19 & $52 \cdot 8$ & 35 & $55 \cdot 8$ \\
\hline Antiplatelet agents & 15 & $51 \cdot \overline{7}$ & 16 & 44.4 & 31 & $47 \cdot 7$ \\
\hline
\end{tabular}

CASCADE, CArdiovaSCulAr Diabetes \& Ethanol; AT, adipose tissue; FPG, fasting plasma glucose; $\mathrm{Hb}_{\mathrm{A} 1 \mathrm{c}}$, glycated Hb; HOMA-IR, homeostatic model assessment of insulin resistance; HDL-C, HDL cholesterol; LDL-C, LDL cholesterol; hs-CRP, high-sensitivity C-reactive protein.

Values are means and standard deviations unless otherwise indicated. Median time from baseline measurements to randomization was $30 \mathrm{~d}$.

${ }^{*}$ Seventy-three participants with type 2 diabetes.

$\dagger P<0.05$ between intervention groups.

Table 2 Two-year changes $(\Delta)$ in abdominal adiposity in the CASCADE sub-study

\begin{tabular}{|c|c|c|c|c|c|c|}
\hline \multirow[b]{2}{*}{ Variable } & \multicolumn{3}{|c|}{ Mineral water ( $n$ 21) } & \multicolumn{3}{|c|}{ Red wine ( $n$ 27) } \\
\hline & $\Delta$ & $95 \% \mathrm{Cl}$ & $P$ value $v$. baseline & $\Delta$ & $95 \% \mathrm{Cl}$ & $P$ value $v$. baseline \\
\hline$\Delta$ weight $(\mathrm{kg})$ & $-1 \cdot 0$ & $-2 \cdot 9,0.9$ & 0.30 & $-1 \cdot 8$ & $-2 \cdot 8,0.2$ & 0.09 \\
\hline$\triangle$ waist circumference $(\mathrm{cm})$ & $-2 \cdot 4$ & $-4 \cdot 5,-0 \cdot 3$ & 0.03 & $-1 \cdot 3$ & $-3 \cdot 6,0.9$ & 0.22 \\
\hline \multicolumn{7}{|l|}{ Absolute AT change $\left(\mathrm{cm}^{2}\right)$} \\
\hline$\Delta$ visceral AT & -87.6 & $-357 \cdot 3,182 \cdot 1$ & 0.50 & $-159 \cdot 4$ & $-491 \cdot 3,172 \cdot 4$ & $\begin{array}{l}0.86 \\
0.33\end{array}$ \\
\hline$\Delta$ subcutaneous AT & $206 \cdot 2$ & $-100 \cdot 2,512 \cdot 8$ & 0.17 & 177.0 & $-162 \cdot 0,516 \cdot 0$ & 0.29 \\
\hline$\Delta$ superficial subcutaneous AT & $62 \cdot 2$ & $-24 \cdot 3,148 \cdot 8$ & 0.15 & -91.8 & $-273 \cdot 7,89 \cdot 0$ & 0.30 \\
\hline$\Delta$ deep subcutaneous AT & 144.0 & $-149 \cdot 6,437.7$ & 0.32 & $226 \cdot 2$ & $-63 \cdot 8,516 \cdot 2$ & 0.12 \\
\hline \multicolumn{7}{|l|}{ Proportional AT change (\%) } \\
\hline$\Delta$ visceral AT & -3.2 & $-8.9,2.5$ & 0.25 & -3.0 & $-8 \cdot 0,2 \cdot 0$ & 0.23 \\
\hline$\Delta$ subcutaneous AT & $3 \cdot 8$ & $-1 \cdot 7,9 \cdot 3$ & $0 \cdot 16$ & 4.8 & $-1 \cdot 1,10 \cdot 7$ & $0 \cdot 11$ \\
\hline$\Delta$ superficial subcutaneous AT & $-0 \cdot 15$ & $-3 \cdot 3,2 \cdot 9$ & 0.92 & -1.9 & $-5 \cdot 0,1 \cdot 2$ & 0.21 \\
\hline$\Delta$ deep subcutaneous AT & 2.9 & $-2 \cdot 8,8.6$ & 0.30 & $5 \cdot 2$ & $-1 \cdot 1,11 \cdot 6$ & 0.10 \\
\hline
\end{tabular}

CASCADE, CArdiovaSCulAr Diabetes \& Ethanol; AT, adipose tissue. 
correlated with $\mathrm{Hb}_{\mathrm{A} 1 \mathrm{c}}$, and the latter is clearly within the same 'pathway'.) When medical treatments used in this patient population were added to the models, the results remained similar (data not shown). As evaluated by the electronic questionnaires filled in by the participants at 0,6 and 24 months, there were no changes in antidiabetic medication use or other medications. In addition, no substantial changes in energy intake $(+126$ (sD 2887) $\mathrm{kJ} / \mathrm{d}$ $(+30 \cdot 2$ (sD 690) kcal/d); $P=0.8$ compared with baseline) or exercise habits $(0.2$ (SD 9.2) MET; $P=0.9$ compared with baseline) were recorded. However, as evaluated by the FFQ, all groups improved their dietary consumption similarly, as expected when adopting a Mediterranean diet ${ }^{(8)}$. When we assessed whether a change in the intake of specific dietary component(s) was associated with fat distribution during the 2-year intervention, we found that 2 -year increases in the intake of PUFA $(r=-0.335, P=0.05)$ and MUFA $(r=-0.372, P=0.03)$ were associated with a 2 -year decrease in VAT. Two-year changes in the consumption of carbohydrates, SFA, protein and fat were not associated with 2-year changes in abdominal adipose tissues.

\section{Discussion}

In the present 2-year randomized controlled intervention trial in patients with type 2 diabetes, we found that moderate red wine consumption, combined with a healthy diet, was not likely to cause a deterioration in the proportion of abdominal adipose fat. Thus, it is possible that long-term moderate wine intake might be safe - with regard to central adiposity and abdominal fat distribution for type 2 diabetics if the wine is consumed as part of a comprehensive healthy dietary intervention.

Some limitations of our study warrant consideration. First, study participants were not blinded to group assignment. Second, although the gold standard for VAT measurement is total VAT volume measured by MRI, we used three MRI slices at L2-L3, L5-L4 and L5-S1 to calculate the area of absolute fat and fat distribution. The rationale for this choice was that the predictive value of VAT area for VAT volume has been shown as an appropriate alternative measure ${ }^{(23-25)}$. Although, to the best of our knowledge, this trial may be considered a relatively large abdominal MRI intervention trial, our sample size might have limited the statistical power to detect moderate differences in the effect of wine $v$. water. In addition, we relied on self-reported alcohol intake to assess adherence, although we did monitor use by keeping track of returned empty bottles. Excluding the participants from the white wine intervention group of the CASCADE study from the current data analysis might have introduced a potential selection bias. Although there are sex differences in the pharmacokinetics of alcohol ${ }^{(26)}$, we provided similar alcohol doses for both sex groups consistent with a conservative and simple protocol for safety issues. One may speculate that a higher wine intake among men might have a greater effect. Also, the higher percentage of men in the wine group, compared with the water group, might have confounded the results. The strengths of the study include its long duration and the use of MRI as a gold standard for abdominal fat imaging. The nutritional education sessions and free mineral water supplied to the water group allowed equal intensity of intervention and enabled assessment of the specific effects of moderate wine consumption within the setting of a Mediterranean diet. The CASCADE design benefited from initiating the intervention in a population that generally has low consumption of alcohol ${ }^{(27)}$ and by reducing the risk for abuse by including participants older than 40 years with a low addictive risk.

Initiation of moderate wine consumption among alcohol-abstaining patients older than 40 years with type 2 diabetes did not promote accumulation of abdominal adiposity. These findings contribute to the discourse on whether alcohol consumption bears risks or benefits to patients with type 2 diabetes. A few studies have reported associations between alcohol consumption and risk of abdominal obesity, as measured by waist circumference, waist-to-hip ratio or computed tomography ${ }^{(1,2,28,29)}$, while others state no association with fat distribution ${ }^{(6)}$ or body weight, reporting a tendency towards a non-linear U-shaped association between changes in waist circumference and wine. A recent study found that an inverse association between alcohol consumption and diabetes was partly explained by fat distribution ${ }^{(30)}$. Although wine and alcohol consumption and its effect on accumulation of abdominal fat are still subject to debate, the current results might clarify some of the uncertainty.

A 2-year decrease in visceral fat was associated with decreased glycaemic control. Growing evidence suggests that VAT accumulation plays an important role in the aetiology of type 2 diabetes $^{(31,32)}$, suggesting that visceral fat may be an important potential target for intervention, especially in patients who present increased visceral $\mathrm{fat}^{(14)}$. In the current analysis, improved $\mathrm{Hb}_{\mathrm{A} 1 \mathrm{c}}$ was independently associated with a decrease in VAT, suggesting that redistribution of fat depots, beyond weight loss, has a positive effect on glycaemic control in persons with type 2 diabetes.

Although not a primary aim of the trial, the 2-year increase in PUFA and MUFA was associated with a reduction in VAT, as has been shown previously; for example, a study of thirty-four men with hypercholesterolaemia, which measured body composition by means of bioelectrical impedance, reported that a diet rich in MUFA decreased total body fat ${ }^{(33)}$. Central body fat accumulation (as measured by dual-energy X-ray absorptiometry) was prevented in eleven insulin-resistant patients ${ }^{(34)}$ who consumed a MUFA-rich diet as opposed to a diet rich in carbohydrates. The Mediterranean dietary guidelines 
given to the participants in the CASCADE study were based on quality rather than quantity of foods, with no emphasis on energy restriction or weight-loss promotion. Our findings imply that the specific increase in MUFA and PUFA as part of a Mediterranean dietary regime may have had an effect on the redistribution of fat depots.

\section{Conclusion}

In summary, the moderate consumption of red wine by patients with type 2 diabetes did not promote abdominal adiposity. To the best of our knowledge, CASCADE is the first large, long-term randomized controlled trial to investigate the effect of alcohol in the diet of patients with type 2 diabetes who had previously been alcohol abstainers. Further intervention studies focusing on specific dietary components and their effect on abdominal fat shift and distribution are recommended.

\section{Acknowledgements}

Acknowledgements: The authors thank the CASCADE participants for their consistent cooperation. They thank Harel Segal from the Nuclear Research Center Negev and Osnat Tangi-Rosental from the Ben-Gurion University of the Negev. Financial support: This work was supported by the European Foundation for the Study of Diabetes. The European Foundation for the Study of Diabetes had no role in the design, analysis or writing of this article. Conflict of interest: The authors have no relationship with the companies that make products relevant to the manuscript. Authorship: R.G. researched data, performed the statistical analyses and wrote the manuscript. I. Shelef performed data analysis, contributed to the discussion, and reviewed and edited the manuscript. E.S., D. Schwarzfuchs, Y.G., S.W., Y.C., D. Serfaty, N.B., M.R., N.O., S.B.-A., I.F.L. and B.S. researched data. I.H.-B. and Y.H. researched data, contributed to the discussion, and reviewed and read the manuscript. M.J.S. contributed to the discussion, and reviewed and read the manuscript. A.R. and I. Shai designed the study, performed data analysis and wrote the manuscript. Ethics of human subject participation: The study was approved and monitored by the Human Subjects Committees of the Soroka University Medical Center and the Ben-Gurion University of the Negev. All participants provided informed consent and received no financial compensation for participation. ClinicalTrials.gov identifier: NCT00784433.

\section{Supplementary material}

To view supplementary material for this article, please visit http://dx.doi.org/10.1017/S1368980016002597

\section{References}

1. Schröder H, Morales-Molina JA, Bermejo S et al. (2007) Relationship of abdominal obesity with alcohol consumption at population scale. Eur J Nutr 46, 369-376.

2. Dallongeville J, Marécaux N, Ducimetière P et al. (1998) Influence of alcohol consumption and various beverages on waist girth and waist-to-hip ratio in a sample of French men and women. Int J Obes Relat Metab Disord 22, 1178-1183.

3. Kimokoti RW, Gona P, Zhu L et al. (2012) Dietary patterns of women are associated with incident abdominal obesity but not metabolic syndrome. J Nutr 142, 1720-1727.

4. Kanerva N, Kaartinen NE, Schwab U et al. (2013) Adherence to the Baltic Sea diet consumed in the Nordic countries is associated with lower abdominal obesity. Br J Nutr $\mathbf{1 0 9}$, 520-528.

5. Greenfield JR, Samaras K, Jenkins AB et al. (2003) Moderate alcohol consumption, dietary fat composition, and abdominal obesity in women: evidence for gene-environment interaction. J Clin Endocrinol Metab 88, 5381-5386.

6. Beulens JW, van Beers RM, Stolk RP et al. (2006) The effect of moderate alcohol consumption on fat distribution and adipocytokines. Obesity (Silver Spring) 14, 60-66.

7. Kim KH, Oh SW, Kwon H et al. (2012) Alcohol consumption and its relation to visceral and subcutaneous adipose tissues in healthy male Koreans. Ann Nutr Metab 60, 52-61.

8. Gepner Y, Golan R, Harman-Boehm I et al. (2015) Effects of initiating moderate alcohol intake on cardiometabolic risk in adults with type 2 diabetes: a 2-year randomized, controlled trial. Ann Intern Med 163, 569-579.

9. Hamdy O, Porramatikul S \& Al-Ozairi E (2006) Metabolic obesity: the paradox between visceral and subcutaneous fat. Curr Diabetes Rev 2, 367-373.

10. Fox CS, Massaro JM, Hoffmann U et al. (2007) Abdominal visceral and subcutaneous adipose tissue compartments: association with metabolic risk factors in the Framingham Heart Study. Circulation 116, 39-48.

11. Matsushita Y, Nakagawa T, Yamamoto S et al. (2010) Associations of visceral and subcutaneous fat areas with the prevalence of metabolic risk factor clustering in 6,292 Japanese individuals: the Hitachi Health Study. Diabetes Care 33, 2117-2119.

12. Scheuer SH, Færch K, Philipsen A et al. (2015) Abdominal fat distribution and cardiovascular risk in men and women with different levels of glucose tolerance. J Clin Endocrinol Metab 100, 3340-3347.

13. Sniderman AD, Bhopal R, Prabhakaran D et al. (2007) Why might South Asians be so susceptible to central obesity and its atherogenic consequences? The adipose tissue overflow hypothesis. Int J Epidemiol 36, 220-225.

14. Gallagher D, Kelley DE, Yim JE et al.; MRI Ancillary Study Group of the Look AHEAD Research Group (2009) Adipose tissue distribution is different in type 2 diabetes. Am J Clin Nutr 89, 807-814.

15. Sam S, Haffner S, Davidson MH et al. (2008) Relationship of abdominal visceral and subcutaneous adipose tissue with lipoprotein particle number and size in type 2 diabetes. Diabetes 57, 2022-2027.

16. Golan R, Shelef I, Rudich A et al. (2012) Abdominal superficial subcutaneous fat: a putative distinct protective fat sub-depot in type 2 diabetes. Diabetes Care 35, 640-647.

17. Shai I, Wainstein J, Harman-Boehm I et al. (2007) Glycemic effects of moderate alcohol intake among patients with type 2 diabetes: a multicenter, randomized, clinical intervention trial. Diabetes Care 30, 3011-3016.

18. Shai I, Shahar DR, Vardi H et al. (2004) Selection of food items for inclusion in a newly developed food-frequency questionnaire. Public Health Nutr 7, 745-749.

19. Shai I, Schwarzfuchs D, Henkin Y et al.; Dietary Intervention Randomized Controlled Trial (DIRECT) Group (2008) 
Weight loss with a low-carbohydrate, Mediterranean, or low-fat diet. $N$ Engl J Med 359, 229-241.

20. So R, Sasai H, Matsuo T et al. (2012) Multiple-slice magnetic resonance imaging can detect visceral adipose tissue reduction more accurately than single-slice imaging. Eur J Clin Nutr 66, 1351-1355.

21. Shen W, Chen J, Gantz M et al. (2012) A single MRI slice does not accurately predict visceral and subcutaneous adipose tissue changes during weight Loss. Obesity (Silver Spring) 20, 2458-2463.

22. Shai I, Rosner BA, Shahar DR et al. (2005) Dietary evaluation and attenuation of relative risk: multiple comparisons between blood and urinary biomarkers, food frequency, and 24-hour recall questionnaires: the DEARR study. J Nutr 135, 573-579.

23. Schwenzer NF, Machann J, Schraml C et al. (2010) Quantitative analysis of adipose tissue in single transverse slices for estimation of volumes of relevant fat tissue compartments: a study in a large cohort of subjects at risk for type 2 diabetes by MRI with comparison to anthropometric data. Invest Radiol 45, 788-794.

24. Demerath EW, Shen W, Lee M et al. (2007) Approximation of total visceral adipose tissue with a single magnetic resonance image. Am J Clin Nutr 85, 362-368.

25. Shen W, Punyanitya M, Wang Z et al. (2004) Total body skeletal muscle and adipose tissue volumes: estimation from a single abdominal cross-sectional image. J Appl Physiol (1985) 97, 2333-2338.

26. Baraona E, Abittan CS, Dohmen K et al. (2001) Gender differences in pharmacokinetics of alcohol. Alcohol Clin Exp Res 25, 502-507.

27. Organisation for Economic Co-operation and Development (2011) Health at a Glance 2011: OECD Indicators.
Paris: OECD Publishing; available at http://dx.doi.org/ 10.1787/health_glance-2011-en

28. Lukasiewicz E, Mennen LI, Bertrais S et al. (2005) Alcohol intake in relation to body mass index and waist-to-hip ratio: the importance of type of alcoholic beverage. Public Health Nutr 8, 315-320.

29. Cigolini M, Targher G, Bergamo Andreis IA et al. (1996) Moderate alcohol consumption and its relation to visceral fat and plasma androgens in healthy women. Int J Obes Relat Metab Disord 20, 206-212.

30. Beulens JW, van der Schouw YT, Bergmann MM et al. (2012) Alcohol consumption and risk of type 2 diabetes in European men and women: influence of beverage type and body size. The EPIC-InterAct study. J Intern Med $\mathbf{2 7 2}$, $358-370$.

31. Gastaldelli A, Miyazaki Y, Pettiti M et al. (2002) Metabolic effects of visceral fat accumulation in type 2 diabetes. J Clin Endocrinol Metab 87, 5098-5103.

32. Kim MK, Jang EH, Son JW et al. (2011) Visceral obesity is a better predictor than generalized obesity for basal insulin requirement at the initiation of insulin therapy in patients with type 2 diabetes. Diabetes Res Clin Pract 93, 174-178

33. Fernández de la Puebla RA, Fuentes F, Pérez-Martinez P et al. (2003) A reduction in dietary saturated fat decreases body fat content in overweight, hypercholesterolemic males. Nutr Metab Cardiovasc Dis 13, 273-277.

34. Paniagua JA, Gallego de la Sacristana A et al. (2007) Monounsaturated fat-rich diet prevents central body fat distribution and decreases postprandial adiponectin expression induced by a carbohydrate-rich diet in insulinresistant subjects. Diabetes Care 30, 1717-1723. 